\title{
Weak Compactness in the Space of Operator Valued Measures and Optimal Control
}

\author{
Nasiruddin Ahmed \\ EECS, University of Ottawa, Ottawa, Canada
}

\begin{abstract}
In this paper we present a brief review of some important results on weak compactness in the space of vector valued measures. We also review some recent results of the author on weak compactness of any set of operator valued measures. These results are then applied to optimal structural feedback control for deterministic systems on infinite dimensional spaces.
\end{abstract}

Keywords: Space of Operator valued measures, Countably additive operator valued measures, Weak compactness, Semigroups of bounded linear operators, Optimal Structural control.

AMS(MOS) Subject Classification: 46A50,46B50,46E27,46E99,47A55.

\section{Introduction}

Necessary and sufficient conditions for weak compactness in the space of vector measures has been a subject of great interest over half a century. One of the seminal results in this topic is the well known Bartle-Dunford-Schwartz theorem 1, Theorem 5, p.105] for countably additive bounded vector measures with values in Banach spaces satisfying, along with their duals, the Radon-Nikodym property. This result was extended to finitely additive vector measures by Brooks [3] and Brooks and Dinculeanu [1, Corollary 6, p.106]. We present in this section some of the celebrated results on this topic. For vector measures see [1] and [2]. First we present the Bartle-Dunford-Schwartz theorem (BDS) 1, Theorem 5, p.105].

Theorem 1.1(BDS). Let $D$ be any set and $\Sigma \equiv \sigma(D)$ denote the sigma algebra of subsets of the set $D$, and $X, X^{*}$ a dual pair of B-spaces satisfying (RadonNikodym Property) RNP. A set $\Gamma \subset M_{c a}(\Sigma, X)$ is relatively weakly compact if, and only if,

(i) $\Gamma$ is bounded

(ii) $\{|\mu|, \mu \in \Gamma\}$ is uniformly c.a

(iii) for each $\sigma \in \Sigma$ the set $\{\mu(\sigma), \mu \in \Gamma\} \subset X$ is weakly relatively compact.

This result was extended to finitely additive (f.a) vector measures by Brooks [3] for reflexive Banach spaces $X$, and then by Brooks and Dinculeanu [1, Corollary $6,106]$ for nonreflexive spaces.

We state here the later result.

D. Hömberg and F. Tröltzsch (Eds.): CSMO 2011, IFIP AICT 391, pp. 49-58, 2013.

(C) IFIP International Federation for Information Processing 2013 
Theorem 1.2 (Brooks \& Dinculeanu). Let $\Sigma$ be an algebra of subsets of a set $D, M_{b a}(\Sigma, X)$ the space of finitely additive vector measures with values in $X$ and $\left\{X, X^{*}\right\}$ satisfy RNP. A set $\Gamma \subset M_{b a}(\Sigma, X)$ is weakly relatively compact if, and only if, the following three conditions are satisfied

(i) $\Gamma$ is bounded

(ii) there exists a f.a nonnegative measure $\nu$ such that $\lim _{\nu(\sigma) \rightarrow 0}|\mu|(\sigma)=0$ uniformly w.r.t $\mu \in \Gamma$.

(iii) for each $\sigma \in \Sigma$, the set $\{\mu(\sigma), \mu \in \Gamma\} \subset X$ is relatively (conditionally) weakly compact.

These results have been used by the author in the study of optimal control of impulsive systems on Banach spaces [13.

Weak sequential compactness for regular vector measures have been studied by Kuo [4, Theorem 1.6, Theorem 3.3] where he gives several results on weak sequential compactness based on set-wise weak convergence.

In physical sciences and engineering, involving control theory and optimization, one has the freedom to choose from a given class of vector or operator valued measures the best one that minimizes or maximizes certain functionals representing a measure of performance of the system. This is where compactness is useful. These problems arise naturally in the area of optimization, optimal control, system identification, Kalman filtering, structural control etc [89|1013]14].

\section{Basic Properties of Operator Valued Measures}

Let $D$ be a compact Hausdorff space and $\Sigma$ an algebra of subsets of $D,\{X, Y\}$ a pair of B-spaces and $\mathcal{L}(X, Y)$ is the space of bounded linear operators from $X$ to $Y$. The function

$$
B: \Sigma \longrightarrow \mathcal{L}(X, Y)
$$

is generally a finitely additive (f.a) set function with values in $\mathcal{L}(X, Y)$. This class, denoted by $M_{b a}(\Sigma, \mathcal{L}(X, Y))$, is called the space of operator valued measures. Clearly, this is a B-space with respect to the topology induced by the supremum of the operator norm on $\Sigma$.

Now we introduce the notion of countable additivity of operator valued measures. Unlike vector measures (Banach space valued), the notion of countable additivity of operator valued measures depends on the topology used for the space of bounded linear operators. Thus if we limit ourselves to the most popular topologies such as uniform, strong, weak operator topologies we have at least three kinds of countable additivity. This is described below.

Definition $2.1\left(c a-\tau_{u o}\right)$ An element $B \in M_{b a}(\Sigma, \mathcal{L}(X, Y))$ is countably additive in the uniform operator topology $\left(c a-\tau_{u o}\right)$ if for any family of pairwise disjoint sets $\left\{\sigma_{i}\right\} \in \Sigma, \sigma_{i} \subset D$ and $\cup \sigma_{i} \in \Sigma$,

$$
\lim _{n \rightarrow \infty}\left\|B\left(\bigcup \sigma_{i}\right)-\sum_{i=1}^{n} B\left(\sigma_{i}\right)\right\|_{\mathcal{L}(X, Y)}=0 .
$$


Similarly we define countable additivity in the strong operator topology as follows.

Definition $2.2\left(c a-\tau_{s o}\right)$ An element $B \in M_{b a}(\Sigma, \mathcal{L}(X, Y))$ is said to be countably additive in the strong operator topology $\left(c a-\tau_{s o}\right)$ if for any family of pair wise disjoint sets $\left\{\sigma_{i}\right\} \in \Sigma, \sigma_{i} \subset D, \cup \sigma_{i} \in \Sigma$, and for every $x \in X$,

$$
\lim _{n \rightarrow \infty}\left|B\left(\bigcup \sigma_{i}\right) x-\sum_{i=1}^{n} B\left(\sigma_{i}\right) x\right|_{Y}=0
$$

Note that if $X=R$ then $B$ reduces to an $Y$ valued vector measure and the countable additivity in the strong operator topology reduces to the usual (norm) countable additivity. Similarly, if $Y=R$ then $B$ reduces to an $X^{*}$-valued vector measure and the countable additivity in the strong operator topology reduces to countable additivity in the weak star topology. One can also define countable additivity in the weak operator topology. Since we do not use it, it is not necessary to include it here.

By Orlicz-Pettis Theorem [12], a vector measure is countably additive if and only if it is weakly countably additive. Thus it follows from this result, that $c a-\tau_{s o} \cong c a-\tau_{w o}$. That is, countable additivity in the strong operator topology is equivalent to countable additivity in the weak operator topology. Next we consider the question of variation. It is known that for Banach space valued vector measures there are two notions of variation, strong variation usually known as variation, and weak variation called semivariation. Again in the case of operator valued measures there are as many possibilities of variations as there are topologies.

For any set $J \in \Sigma$, let us denote the class of finite disjoint $\Sigma$ measurable partitions of $J$ by $\Pi_{\Sigma}(J)$.

Definition 2.3- $\tau_{u o}$ :(Variation in the $\left.\tau_{u o}\right)$ For any $B \in M_{b a}(\Sigma, \mathcal{L}(X, Y))$ its variation in the uniform operator topology on the set $J$ is given by

$$
|B|_{u}(J)=\sup _{\pi \in \Pi_{\Sigma}(J)} \sum_{\sigma \in \pi}\|B(\sigma)\|_{\mathcal{L}(X, Y)}
$$

where the supremum is taken over $\Pi_{\Sigma}(J)$.

Clearly, if $X=R$ then the operator valued measure $B$ reduces to an $Y$-valued vector measure and the above expression gives the standard variation of vector measures.

Definition 2.4- $\tau_{s o}$ (Variation in the $\tau_{s o}$ ). The variation of $B$ on $J$ in the strong operator topology is given by:

$$
|B|_{s}(J)=\sup \left\{\left|\sum_{i=1}^{n} B\left(\sigma_{i}\right) x_{i}\right|_{Y}, x_{i} \in B_{1}(X),\left\{\sigma_{i}, 1 \leq i \leq n,\right\} \in \Pi_{\Sigma}(J), n \in N\right\} .
$$

Again if $X=R$ then $B$ reduces to an $Y$ valued vector measure and the above expression gives the standard semivariation of vector measures. Similarly one can define variation in the weak operator topology. 
The uniform, strong, and weak variations of $B$ over $D$ are given respectively by

$$
\begin{gathered}
|B|_{u}(D) \equiv \sup \left\{|B|_{u}(\sigma), \sigma \in \Sigma\right\}, \quad|B|_{s}(D) \equiv \sup \left\{|B|_{s}(\sigma), \sigma \in \Sigma\right\}, \quad \text { and } \\
|B|_{w}(D) \equiv \sup \left\{|B|_{w}(\sigma), \sigma \in \Sigma\right\}
\end{gathered}
$$

It is easy to verify that $|B|_{w} \leq|B|_{s} \leq|B|_{u}$. Clearly, this result means that an element $B \in M_{b a}(\Sigma, \mathcal{L}(X, Y))$ may have finite strong variation while it has infinite uniform variation. That is , $|B|_{s}<\infty$ but $|B|_{u}=\infty$.

Let $B_{\infty}(D, X)$ denote the vector space of bounded $\Sigma$-measurable $X$ valued functions which are uniform limits of $\Sigma$-measurable simple functions $\mathcal{S}(D, X)$. Endowed with sup norm topology, $B_{\infty}(D, X)$ is a B-Space. Let $\mathcal{L}_{1}(X, Y)$ denote the B-space of nuclear operators. It is well known that the Grothendieck characterization of $L \in \mathcal{L}_{1}(X, Y)$ is given by

$$
L x \equiv \sum \lambda_{i} x_{i}^{*}(x) y_{i}, x_{i}^{*} \in \partial B_{1}\left(X^{*}\right), y_{i} \in \partial B_{1}(Y)
$$

with $\sum\left|\lambda_{i}\right|<\infty$, where $\left\{x_{i}, x_{i}^{*}\right\} \in X \times X^{*},\left\{y_{i}, y_{i}^{*}\right\} \in Y \times Y^{*}$ are the normalized bi-orthogonal basis of the spaces $X$ and $Y$, respectively.

\section{$3 \quad$ Weak Compactness}

Now we can present some recent results on the characterization of conditionally weakly compact sets in the space of operator valued measures. The first result presented here involves Hilbert spaces and nuclear operator valued measures.

Theorem 3.1. Let $\{X, Y\}$ be a pair of separable Hilbert spaces with complete ortho-normal basis $\left\{x_{i}, y_{i}\right\}$. A set $\Gamma \subset M_{b a}\left(\Sigma, \mathcal{L}_{1}(X, Y)\right)$ is conditionally weakly compact if, and only if, the following conditions hold:

(c1): $\Gamma$ is bounded,

(c2): for each $\sigma \in \Sigma, \sum_{i=1}^{\infty}\left|\left(M(\sigma) x_{i}, y_{i}\right)_{Y}\right|$ is convergent uniformly with respect to $M \in \Gamma$,

(c3): for each $i \in N$, the set of scalar valued measures $\left\{\mu_{M}(\cdot)=\left(M(\cdot) x_{i}, y_{i}\right)\right.$, $M \in \Gamma\}$ is a conditionally weakly compact subset of $M_{b a}(\Sigma)$.

Proof. [10, Theorem 3.2, PMD,(2010),p1-15]

This result was recently extended to more general spaces of operator valued measures. Here we consider $\{X, Y\}$ to be a pair of Banach spaces and replace the space of nuclear operators by $\mathcal{L}(X, Y)$, the space of bounded linear operators. Let

$$
M_{\text {casbsv }}(\Sigma, \mathcal{L}(X, Y)) \subset M_{b a}(\Sigma, \mathcal{L}(X, Y))
$$

denote the space of operator valued measures countably additive in the strong operator topology having bounded semivariations (variation in the strong operator topology). To proceed further, we need to consider the question of integration 
of vector valued functions with respect to operator valued measures. The most general theory of integration was introduced by Dobrakov [56]. This generalizes the theory of Lebesgue integral, Bochner integral, Bartle bilinear integral and Dinculeanu integral etc.

For convenience of the reader we recall that a formal series $\sum x_{n}, x_{n} \in X$, is said to be unconditionally convergent if $\sum x_{\pi(n)}$ is convergent for every permutation $\pi: N \rightarrow N$. Orlicz-Pettis Theorem [1, Corollary 4, p.22] states that if every subseries of the series is weakly convergent then the series is unconditionally strongly convergent. This is the foundation of Dobrakov integral.

Dobrakov Integral: For any $f \in B_{\infty}(D, X)$ and $T \in M_{\text {casbsv }}(\Sigma, \mathcal{L}(X, Y))$ the integral,

$$
I_{T}(f) \equiv \int_{D} T(d s) f(s) \in Y,
$$

is well defined in the sense of Dobrakov [5]. As usual the integral is first defined for simple functions $\mathcal{S}(D, X)$ and then extended to $B_{\infty}(D, X)$ by density argument. The most important point is that the limit is taken in the sense of unconditional convergence of the sum arising from the simple functions. This limit is the Dobrakov integral. This is unlike the Lebesgue and Bochner integrals which are based on absolute convergence. This is where the main difference is.

Now we can introduce the notion of Dobrakov semivariation as follows.

Definition 3.2. (Dobrakov semivariation) For any $T \in M_{\text {casbsv }}(\Sigma, \mathcal{L}(X, Y))$ and $\sigma \in \Sigma$ define the set function given by

$$
\hat{T}(\sigma) \equiv \sup \left\{\left|\int_{\sigma} T(d s) f(s)\right|_{Y}, f \in \mathcal{S}(D, X),\|f\|_{\infty} \leq 1\right\} .
$$

Then the Dobrakov semivariation of $T$ over $D$ is given by $\hat{T}(D) \equiv \sup \{\hat{T}(\sigma)$, $\sigma \in \Sigma\}$.

The reader can easily verify that $\hat{T}(D)=|T|_{s}$. In other words, Dobrakov semi varition is the same as the variation in the strong operator topology.

We need few more concepts before we can return to the compactness issue.

Definition 3.3 (F-Space): A compact Hausdorff space $D$ is said to be an $\mathcal{F}$-space if every pair of disjoint open $F_{\sigma}$ set has disjoint closure, [4, Kuo].

Definition 3.4(Grothendieck Space): A Banach space $X$ is said to be a Grothendieck space if weak star convergence in its dual $X^{*}$ is equivalent to weak convergence. In other words the $X$ topology of $X^{*}$ is equivalent to the $X^{* *}$ topology of $X^{*}$.

Well known examples of Grothendieck spaces are reflexive Banach space and separable dual spaces. For more examples see Diestel \& Uhl [1]. Another characterization of Grothedieck space $X$ is that, for every separable Banach space 
$Y$, every bounded linear operator from $X$ to $Y$ is weakly compact. If $K$ is a compact metric space then $C(K)$, the space of continuous functions on $K$, is a Grothendieck space. The space $L_{\infty}(\mu)$ is a Grothendieck space if $\mu$ is a positive measure.

A General Result on Weak Compactness: Now we are prepared to present a general result characterizing weakly compact sets in $M_{\text {casbsv }}(\Sigma, \mathcal{L}(X, Y))$. Let $\Gamma \subset M_{\text {casbsv }}(\Sigma, \mathcal{L}(X, Y))$ and $f \in B_{\infty}(D, X)$. Define the set

$$
\Gamma(f) \equiv\left\{\mu \in M_{b a}(\Sigma, Y): \mu(\sigma)=\int_{\sigma} T(d s) f(s), \sigma \in \Sigma, T \in \Gamma\right\} .
$$

It is easy to verify that $\Gamma(f) \subset M_{c a}(\Sigma, Y) \subset M_{b a}(\Sigma, Y)$.

Theorem 3.5. Suppose $D$ is a compact Hausdorff $\mathcal{F}$-space, and $\{X, Y\}$ is a pair of B-spaces with $Y$ being reflexive. Then a set $\Gamma \subset M_{\text {casbsv }}(\Sigma, \mathcal{L}(X, Y))$ is conditionally weakly compact if, and only if, the following conditions hold:

(i): $\Gamma$ is bounded in the sense that $\sup \left\{\hat{T}(D) \equiv|T|_{s}, T \in \Gamma\right\}<\infty$.

(ii): For each $f \in B_{\infty}(D, X)$, the set $\{|\mu|(\cdot), \mu \in \Gamma(f)\}$ is uniformly c.a.

Proof. Detailed proof appears in Ahmed [11], Theorem 1]. Here we present only a brief outline. Since $\Gamma$ is bounded, for each $f \in B_{\infty}(D, X)$, the set $\Gamma(f)$ is a bounded subset of $M_{c a}(\Sigma, Y)$. By hypothesis (ii), $\Gamma(f)$ is uniformly countably additive. Thus, since $Y$ is reflexive, it follows from Brooks theorem [3, Main Theorem, Cor.1, p284] that for each $f \in B_{\infty}(D, X)$, the set $\Gamma(f)$ is a conditionally weakly compact subset of $M_{c a}(\Sigma, Y)$. Rest of the proof presents arguments to demonstrate that this implies conditional weak compactness of $\Gamma$ itself and conversely. The tools used are: a result of Kuo [4] that asserts that if $D$ is an $F$-space and $Y$ is reflexive then $C\left(D, Y^{*}\right)$ is a Grothedieck space. Thus $w^{*}$ and weak convergence in $M_{c a}(\Sigma, Y)$ are equivalent. Next we use Nikodym uniform boundedness principle [1, Theorem 1, p14] and the classical uniform boundedness principle for linear operators. Then we use the fact that any closed bounded convex subset of $\mathcal{L}(X, Y)$ is compact in the weak operator topology if and only if $Y$ is reflexive. We also use Hahn-Banach theorem to prove boundedness of the semivariation of the limit of any convergent sequence from $\Gamma$. Finally to prove countable additivity in the strong operator topology, we use Pettis theorem which states that a weakly countably additive vector measure defined on a sigma algebra is (strongly) countably additive. This completes the outline of our proof.

\section{Some Remarks and Open Problems}

(R1): Note that we have characterized conditionally weakly compact sets in the space $M_{\text {casbsv }}(\Sigma, \mathcal{L}(X, Y))$. This is a subspace of the space of finitely additive operator valued measures having finite semivariations denoted by $M_{\text {fabsv }}(\Sigma,(X, Y))$. The author believes that following our approach one can obtain characterization of conditional weak compactness in this space also. In any case it would be interesting to characterize weakly compact sets in the two larger spaces: 


$$
M_{\text {fabsv }}(\Sigma, \mathcal{L}(X, Y)) \subset M_{b a}(\Sigma, \mathcal{L}(X, Y))
$$

(R2): Another fact that we have used in the proof of the above theorem is that the closed unit ball $B_{1}(\mathcal{L}(X, Y))$ is compact in the weak operator topology. This means that $Y$ is reflexive. In fact this is a necessary and sufficient condition for weak compactness of $B_{1}(\mathcal{L}(X, Y))$. It would be interesting to extend our result to cases where $Y$ is not necessarily reflexive. In other words to improve our result one must avoid using the compactness of $B_{1}(\mathcal{L}(X, Y))$ in the weak operator topology.

(R3): One of the very important topic in vector measure theory is the representation theory like the Riesz representation theorem. By virtue of Dobrakov theory, every

$$
T \in M_{\text {casbsv }}(\Sigma, \mathcal{L}(X, Y))
$$

determines a linear operator $L_{T} \in \mathcal{L}\left(B_{\infty}(D, X), Y\right)$, the space of bounded linear operators from $B_{\infty}(D, X)$ to $Y$, satisfying $\left\|L_{T}\right\|=|T|_{s}$, and so we have the embedding

$$
M_{\text {casbsv }}(\Sigma, \mathcal{L}(X, Y)) \hookrightarrow \mathcal{L}\left(B_{\infty}(D, X), Y\right) .
$$

The question is: does every $L \in \mathcal{L}\left(B_{\infty}(D, X), Y\right)$ have the integral representation with some $T \in M_{\text {casbsv }}(\Sigma, \mathcal{L}(X, Y))$. The answer seems to be no. Note that every $T \in M_{\text {fabsv }}(\Sigma, \mathcal{L}(X, Y))$ determines a continuous linear operator $L_{T}$ on $B_{\infty}(D, X)$ to $Y$ through the Dobrakov integral

$$
L_{T}(f)=\int_{D} T(d s) f(s) .
$$

This follows from the fact that

$$
\left|L_{T}(f)\right|_{Y}=\left|\int_{D} T(d s) f(s)\right|_{Y} \leq|T|_{s}\|f\|_{B_{\infty}(D, X)} .
$$

This also shows that $M_{\text {fabsv }}(\Sigma, \mathcal{L}(X, Y)) \hookrightarrow \mathcal{L}\left(B_{\infty}(D, X), Y\right)$. In fact we can prove that

$$
M_{\text {fabsv }}(\Sigma, \mathcal{L}(X, Y)) \cong \mathcal{L}\left(B_{\infty}(D, X), Y\right)
$$

(R4): Since $Y$ is a reflexive Banach space, every operator $L \in \mathcal{L}\left(B_{\infty}(D, X), Y\right)$ is weakly compact in the sense that it maps any bounded subset of $B_{\infty}(D, X)$ into a relatively weakly compact subset of $Y$. Also for the same reason the closed unit ball $\mathcal{B}_{1}\left(\mathcal{L}\left(B_{\infty}(D, X), Y\right)\right)$ is compact in the weak operator topology. Thus every net $\left\{L_{\alpha}, \alpha \in \Lambda\right\} \in \mathcal{B}_{1}$ has a subnet that converges in the weak operator topology to some $L_{o} \in \mathcal{B}_{1}\left(\mathcal{L}\left(B_{\infty}(D, X), Y\right)\right)$. If the net $\left\{L_{\alpha}, \alpha \in \Lambda\right\}$ were generated by a net $B_{\alpha} \in M_{\text {casbsv }}(\Sigma, \mathcal{L}(X, Y))$, the limit operator $L_{o}$ may not be represented by a measure $B_{o}$ from $M_{\text {casbsv }}(\Sigma, \mathcal{L}(X, Y))$. In view of the isomorphism stated above, the representing measure $B_{o}$ corresponding to $L_{o}$ may very well be an element of $M_{\text {fabsv }}(\Sigma, \mathcal{L}(X, Y))$. 


\section{Applications to Optimal Feedback Control}

In this section we present two applications of weak compactness to structural control problems in infinite dimension involving deterministic systems. The result also applies to stochastic systems on Hilbert spaces.

Consider the structural control system on a real Banach space $X$

$$
\begin{aligned}
& d x=A x d t+B(d t) y+f(x) d t, x(0)=\xi \\
& y=L x+\eta \quad \text { (output) }
\end{aligned}
$$

over the time interval $t \in[0, T]$. The state space $X$ is a reflexive $B$-space and the output space $Y$ is any real Banach space. The operator $L \in \mathcal{L}(X, Y)$ represents the sensor and $\eta$ is a deterministic bounded $Y$ valued perturbation. The objective functional is given by

$$
J(B) \equiv \int_{0}^{T} \ell(t, x(t)) d t+|B|_{s},
$$

where $|B|_{s}$ denotes the semivariation of $B$ over the set $I$. The admissible set of structural controls is given by a set $\Gamma \subset M_{\text {casbsv }}\left(\Sigma_{I}, \mathcal{L}(Y, X)\right)$. The objective is to find a control that minimizes this functional.

Let $\mathcal{G}_{0}(M, \omega)$ denote the class of infinitesimal generators $\{A\}$ of $C_{0}$-semigroups of linear operators on $X$ with stability parameters $(M, \omega)$ for $M \geq 1$ and $\omega \in R$.

Theorem 4.1. Suppose $A \in \mathcal{G}_{0}(M, \omega)$ generating the semigroup $S(t), t \geq 0$, compact for $t>0, \Gamma$ a weakly compact subset of $M_{\text {casbsv }}\left(\Sigma_{I}, \mathcal{L}(Y, X)\right), f$ locally Lipschitz with at most linear growth, $L \in \mathcal{L}(X, Y), \eta \in B_{\infty}(I, Y)$. There exists $\nu \in M_{c a b v}^{+}\left(\Sigma_{I}\right)$ such that $|B|_{s}(\sigma) \leq \nu(\sigma)$ for $\sigma \in \Sigma_{I}$ uniformly w.r.t $B \in \Gamma$. The cost integrand $\ell$ is measurable in $t$ and lower semicontinuous in $x$ on $X$ and there exists $\alpha \in L_{1}^{+}(I)$ and $\beta \geq 0$ satisfying

$$
|\ell(t, x)| \leq \alpha(t)+\beta|x|_{X}^{p} \text {, for any } p \in(0, \infty) .
$$

Then, there exists a $B_{o} \in \Gamma$ at which $J$ attains its minimum.

Proof. For detailed proof see [11, Theorem 1]. We present a brief outline. For existence and uniqueness of (mild) solutions of the system the reader may see [8, Theorem 3.5, p106]. According to this theorem, the mild solutions are elements of $B_{\infty}(I, X)$. We concentrate on the question of existence of optimal controls. For $\xi \in X$, and $B \in \Gamma$, let $x(B)(\cdot) \in B_{\infty}(I, X)$ denote the mild solution of the system (11) (2). Under the given assumptions, it is easy to verify that there exists a ball $B_{r} \subset X, r \in(0, \infty)$, such that $x(B)(t) \in B_{r}$ for all $t \in I$ and all $B \in \Gamma$. Since the set $\Gamma \subset M_{\text {casbsv }}\left(\Sigma_{I}, \mathcal{L}(Y, X)\right)$ is weakly compact, it suffices to prove that $B \longrightarrow J(B)$ is weakly lower semicontinuous. Let $B_{n} \stackrel{w}{\longrightarrow} B_{o}$ in $M_{\text {casbsv }}\left(\Sigma_{I}, \mathcal{L}(Y, X)\right)$ and let $\left\{x_{n}, x_{o}\right\}$ denote the corresponding mild solutions of the output feedback system

$$
\begin{aligned}
& d x=A x d t+B(d t) L x+f(x) d t+B(d t) \eta(t), \\
& x(0)=\xi,
\end{aligned}
$$


corresponding to $B=B_{n}$ and $B=B_{o}$, respectively. Using compactness property of the semigroup $S(t), t>0$, and a generalized Gronwall type inequality [12] relative to a nonnegative countably additive measure, we show that $x_{n}(t) \stackrel{s}{\longrightarrow}$ $x_{o}(t)$ in $X$ for each $t \in I$. Then it follows from lower semicontinuity of $\ell$ in the second argument that

$$
\ell\left(t, x_{o}(t)\right) \leq \liminf \ell\left(t, x_{n}(t)\right) \text { a.a } t \in I .
$$

By use of Hahn-Banach theorem and weak convergence of $B_{n}$ to $B_{o}$ in the space $M_{\text {casbsv }}\left(\Sigma_{I}, \mathcal{L}(Y, X)\right)$, one can verify without any difficulty that $\left|B_{o}\right|_{s} \leq$ $\lim \inf \left|B_{n}\right|_{s}$. By assumption on $f$ and $\ell$, and $\{\alpha, \beta, p\}$, we have $\ell\left(\cdot, x_{o}(\cdot)\right) \in L_{1}(I)$ implying $J(B)>-\infty$ for all $B \in \Gamma$. Using these results and Fatou's lemma, we obtain

$$
J\left(B_{o}\right) \leq \liminf J\left(B_{n}\right) .
$$

This shows that $B \longrightarrow J(B)$ is w.l.s.c on $M_{\text {casbsv }}\left(\Sigma_{I}, \mathcal{L}(Y, X)\right)$ and bounded away from $-\infty$. Since by assumption $\Gamma$ is compact with respect to the weak topology, $J$ attains its minimum on $\Gamma$.

Remark. Assumption on compactness of the semigroup $S(t)$ can be relaxed by imposing a stronger assumption on the admissible set $\Gamma$. For example, one may assume that $\Gamma$ is compact in the sense that any sequence $B_{n} \in \Gamma$ has a subsequence that converges in the strong operator topology set-wise on $\Sigma$ to an element of $\Gamma$. That is, there exists a $B_{o} \in \Gamma$ such for each $\sigma \in \Sigma, B_{n_{k}}(\sigma) \stackrel{\tau_{s o}}{\longrightarrow}$ $B_{o}(\sigma)$ in $\mathcal{L}(X, Y)$.

Time Optimal Control. Given the initial state $\xi \in X$ and a nonempty closed target set $C \subset X$ not containing $\xi$, the problem is to find a control $B \in \Gamma$ that drives the system to $C$ in minimum time. Since the solutions $\left\{x^{B}, B \in \Gamma\right\}$ are elements of $B_{\infty}(I, X)$, and so not necessarily continuous, we must formulate the objective functional as follows:

$$
J(B) \equiv \inf \left\{t \geq 0: \int_{0}^{t} I_{C}\left(x^{B}(s)\right) d s>\varepsilon\right\},
$$

where $I_{C}(x)$ is the characteristic function of the set $C$ taking value 1 for $x \in C$ and 0 outside $C$ and $\varepsilon \in(0,1)$. Note that $\varepsilon>0$ can chosen as small as necessary. We use the convention that $\inf (\emptyset)=+\infty$. The problem is to find $B_{o} \in \Gamma$ so that $J\left(B_{o}\right) \leq J(B)$ for all $B \in \Gamma$.

Theorem 4.2. Suppose the assumptions of Theorem 4.1 related to $\{A, f, L, \eta, \Gamma\}$ hold. Consider the output feedback system (44) and the time optimal control problem as stated above with the target set $C$, a nonempty closed subset of $X$, not containing $\xi$. Suppose there exists at least one $B \in \Gamma$ for which the set $\left\{t \geq 0: \int_{0}^{t} I_{C}\left(x^{B}(s)\right) d s>\varepsilon\right\} \neq \emptyset$. Then there exists a time optimal control.

Proof. For lack of space we can only present a brief outline. Since $C$ is a closed set the characteristic function $I_{C}$ is upper semicontinuous on $X$. Using this fact 
we prove that the map $B \longrightarrow J(B)$ given by the expression (5) is weakly lower semicontinuous on $\Gamma$ and since this set is weakly compact $J$ attains its minimum on $\Gamma$. Thus time optimal control exists.

\section{References}

1. Diestel, J., Uhl Jr., J.J.: Vector Measures. American Mathematical Society, Providence (1977)

2. Dunford, N., Schwartz, J.T.: Linear Operators, Part 1, General Theory, Second Printing (1964)

3. Brooks, J.K.: Weak Compactness in the Space of Vector Measures. Bulletin of the American Mathematical Society 78(2), 284-287 (1972)

4. Kuo, T.: Weak Convergence of Vector Measures on F-Spaces. Math. Z. 143, 175$180(1975)$

5. Dobrakov, I.: On integration in Banach spaces I. Czechoslovak Mathematical Journal 20(95), 511-536 (1970)

6. Dobrakov, I.: On Integration in Banach Spaces IV. Czechoslovak Mathematical Journal 30(105), 259-279 (1980)

7. Brooks, J.K., Lewis, P.W.: Linear Operators and Vector Measures. Trans. American Math. Soc. 192, 139-162 (1974)

8. Ahmed, N.U.: Vector and Operator Valued Measures as Controls for Infinite Dimensional Systems: Optimal Control. Differential Inclusions, Control and Optimization 28, 95-131 (2008)

9. Ahmed, N.U.: Impulsive Perturbation of $C_{0}$-Semigroups by Operator Valued Measures. Nonlinear Funct. Anal. \& Appl. 9(1), 127-147 (2004)

10. Ahmed, N.U.: Weak Compactness in the Space of Operator Valued Measures. Publicationes Mathematicae, Debrechen (PMD) 77(3-4), 399-413 (2010)

11. Ahmed, N.U.: Weak Compactness in the Space of Operator Valued Measures $M_{b a}(\Sigma, \mathcal{L}(X, Y))$ and its Applications. Differential Inclusions, Control and Optimization 31, 231-247 (2011)

12. Ahmed, N.U.: Some Remarks on the Dynamics of Impulsive Systems in Banach Spaces. Dynamics of Continuous, Discrete and Impulsive Systems 8, 261-274 (2001)

13. Ahmed, N.U.: Existence of Optimal Controls for a General Class of Impulsive Systems on Banach Spaces. SIAM J. Control. Optim. 42(2), 669-685 (2003)

14. Ahmed, N.U.: Dynamics of Hybrid systems Induced by Operator Valued Measures. Nonlinear Analysis: Hybrid Systems 2, 359-367 (2008) 